\title{
Dendritic polyglycerols are modulators of microglia-astrocyte crosstalk
}

Dusica Maysinger* ${ }^{\star}, 1$, Mélanie Lalancette-Hébert ${ }^{\ddagger}{ }^{\ddagger 2}$, Jeff $\mathrm{Ji}^{1}$, Katherine Jabbour ${ }^{1}$, Jens

Dernedde $^{3}$ (D), Kim Silberreis ${ }^{3,4}$, Rainer Haag ${ }^{4}$ \& Jasna Kriz²

${ }^{1}$ Department of Pharmacology \& Therapeutics, McGill University, Montreal, Canada, H3G 1Y6

${ }^{2}$ Department of Psychiatry \& Neuroscience, Laval University, Quebec, Canada, G1J 2G3

${ }^{3}$ Klinische Chemie und Pathobiochemie, Charité-Universitätsmedizin, Berlin Institute of Health, Berlin, Germany

${ }^{4}$ Chemie und Biochemie, Freie Universität Berlin, Berlin, Germany

*Author for correspondence: Tel.: +514 398 1264; dusica.maysinger@mcgill.ca

$\ddagger$ Authors contributed equally

Aim: To determine the ability of sulfated dendritic polyglycerols (dPGS) to modulate neuroglia activation challenged with lipopolysaccharide (LPS). Materials \& methods: Microglia/astrocyte activation in vivo was determined in transgenic animals expressing TLR2-/GFAP-luciferase reporter. Mechanisms implicated in microglia-astrocyte crosstalk were studied in primary mouse brain cultures. Results \& discussion: dPGS significantly reduced microglia activation in vivo, and decreased astrocytic LCN2 production. Activated microglia are necessary for astrocyte stimulation and increase in LCN2 abundance. LCN2 production in astrocytes involves signaling via toll-like receptor 4, activation of NF-кB, IL6 and enhancement of reactive oxygen species. Conclusion: dPGS are powerful modulators of microglia-astrocyte crosstalk and LCN2 abundance; dPGS are promising anti-inflammatory dendritic nanostructures.

\section{Graphical abstract:}

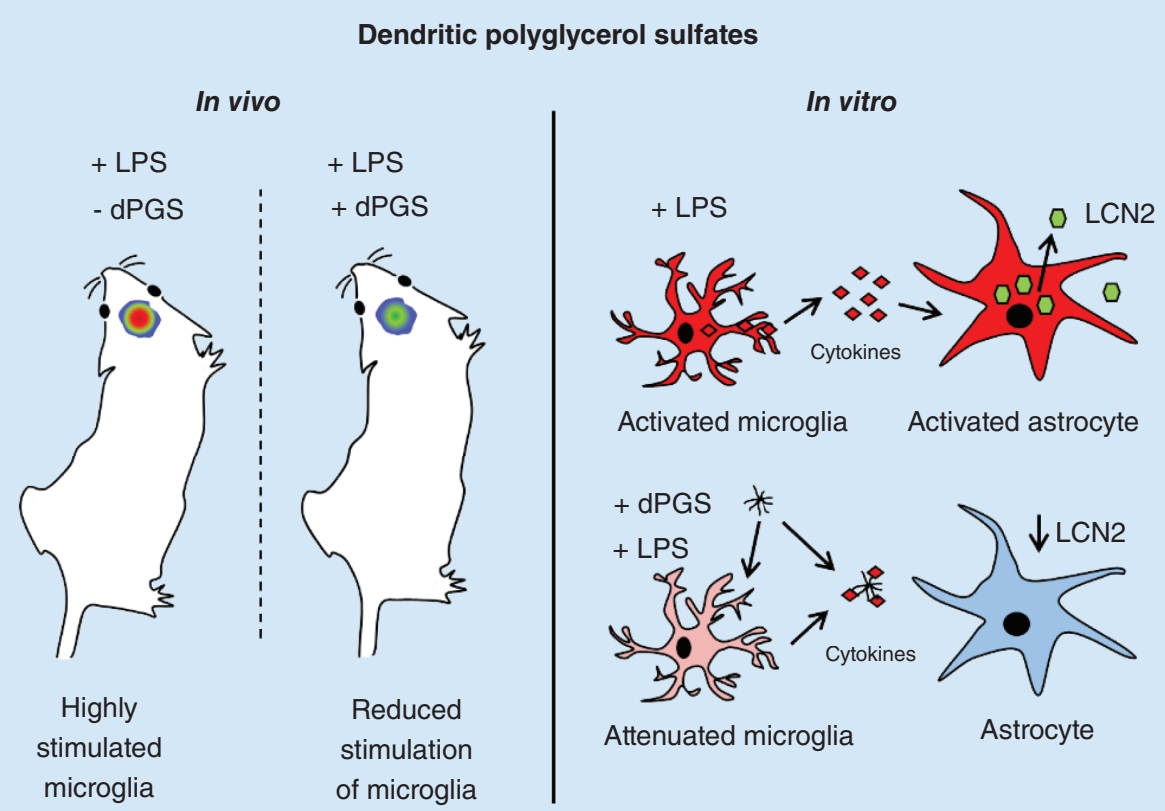

Future Medicine 
Lay abstract: We investigated dendritic polymer effects on brain glial cells: microglia and astrocytes. Microglia are immune cells of the CNS that modulate the activity of astrocytes and production of destructive or protective proteins, including lipocalin-2. We tested dendritic polymers with sulfate terminal groups in both animal models and cultured primary brain cells. We show that a sulfate end group is necessary for polyglycerol dendrimers to exert anti-inflammatory activity and reduce lipocalin-2 production in astrocytes. Sulfated dendritic polyglycerols merit investigations as nanotherapeutics in neurodegenerative disorders associated with inflammation in the brain.

First draft submitted: 2 May 2019; Accepted for publication: 15 August 2019; Published online: 9 October 2019

Keywords: astrocytes $\bullet$ dPGS $\bullet$ IL6 $\bullet$ inflammation $\bullet$ lipocalin-2 $\bullet$ live imaging $\bullet$ LPS $\bullet$ microglia $\bullet$ neuroinflammation

A recent progress in nanotechnology includes the development and use of dendrimers, a globular and hyperbranched type of nanostructure, some of which exert anti-inflammatory effects [1-3]. Dendrimers are synthetic molecules made through a sequential process and possess a central core, branch units and a shell with terminal functional groups [4,5]. Their nanometric size and multivalency enable them to effectively interact with their biological targets [6]. Depending on the size, structure and functional groups, dendrimers can be used for diverse biological applications [7]. Dendrimers have been used for drug and gene delivery and tested in different disease models [8,9]. A generation 3.5 PAMAM dendrimer was shown to reduce the release of pro-inflammatory cytokines from macrophages treated with lipopolysaccharide (LPS) [10], suggesting that some of them have intrinsic anti-inflammatory effects [5,11,12]. Findings from our group [3] also showed that dendritic polyglycerol sulfates exert strong anti-inflammatory effects in cultured neural cells in 2D and 3D [13]. The goal of this study was to investigate the immunomodulatory effect of sulfated and nonsulfated dendritic polyglycerols on neuroglia, stimulated with lipopolysaccharide, a Gram-negative bacterial endotoxin, commonly used as a neuroglia activator. Purity of nanoparticle (NP) is an important issue in biological investigations and thorough screening is necessary [14].

LCN2, also known as neutrophil gelatinase-associated lipocalin, is a member of the lipocalin family of proteins containing a binding domain for small hydrophobic molecules [15]. LCN2 is upregulated in cells exposed to stressful stimuli, ranging from ischemic stroke to infection-induced neuroinflammation and associated redox disturbances [16,17]. LCN2 is expressed in different cell types including those in the CNS [18]. It binds to two kinds of surface receptors, the brain type organic cation transporter $(24 \mathrm{p} 3 \mathrm{R})$ and megalin. LCN2 regulates cellular processes such as cell migration, cell morphology, phenotype polarization, cell death and survival, and more notably iron transport $[15,16,19]$. Protective and destructive roles of LCN2 have both been reported depending on the cell type, duration of exposure and insult inducer [20]. A well-known LCN2 inducer is LPS [20]. LCN2 regulation by dendritic polyglycerols in vivo and the mechanism of their modulatory effects in microglia and astrocytes exposed to LPS has not been reported [21-24]. Evidence from our recent studies suggest that following an acute LPS challenge microglia robustly upregulate $L C N 2$ [25]. Interestingly, the translation of $L C N 2$ transcripts is blocked in LPS-stimulated microglia [25]. Since LCN2 protein is upregulated in the brain upon innate immune challenge and subsequent microglia activation, cell types other than microglia must be source of LCN2 production.

Microglia are sensitive and rapidly responding 'sensors' for pro-inflammatory stimuli. They can take on a particular phenotype based on their microenvironment, as well as the intensity, duration and the type of insult [26]. These phenotypes may involve the secretion of pro-inflammatory cytokines that can in turn activate astrocytes [27]. Reactive astrocytes release both pro- and anti-inflammatory factors and the balance between these diverse endogenous compounds determines their own fate and those of neurons [28,29]. Earlier studies have shown that some nanostructured materials cause cytokine upregulation and release from macrophages [14], but the status of LCN2 in the CNS macrophages, in other words, microglia, and astrocytes upon polyglycerol exposure has not been investigated.

Considering that microglia communicate with astrocytes and neurons, we employed in vitro and in vivo models which provide heterogeneous intercellular interactions. We used LPS to stimulate microglia and astrocytes in vivo in transgenic animals expressing luciferase driven by TLR2 and GFAP promoters. As previously shown, the advantage of these in vivo model-systems is that activation of glial cells (microglia and astrocytes), in response to LPS and nanoparticles can be visualized longitudinally from the brains of live animals using a high-resolution high sensitivity CCD camera and bioluminescence imaging [30-33]. Here, we analyzed in vivo response of microglia and astrocytes to LPS and assessed potential immune-modulatory effects of nonsulfated and sulfated dendritic polyglycerols. 
Intranasal administration of LPS and dendritic polyglycerols was selected to avoid any mechanical insults which could contribute to glial cells stimulation. We show several signaling steps involved in LCN2 protein production and we propose that the modulatory effects of LCN2 regulation in astrocytes require microglia activation.

\section{Materials \& methods}

\section{Transgenic mice \& genotyping}

TLR2-luc-GFP transgenic mice were used to visualize microglial cell activation as described before [30,3335]. The presence of the luciferase transgene was detected by PCR with the subsequent primers: 5'CAGCAGGATGCTCTCCAGTTC-3' and 5'GGCGCAGTAGGCAAGGTGGT3' following the previously described protocol [30]. GFAP-fluc transgenic mice were used to visualize astrocyte activation as previously described [36]. The mice were genotyped by PCR using 5'GAAATGTCCGTTCGGTTGGCAGAAGC and 5' CCAAAACCGTGATGGAATGGAACAACA as primers following the previously described protocol [36]. All experimental procedures were approved by the Université Laval Animal Care Ethics Committee (protocol \#14-096-4), the Ethics Committee for Animal Research at McGill University (protocol \#2008-5521), and are in accordance with the Guide to the Care and Use of Experimental Animals of the Canadian Council on Animal Care.

\section{Intranasal injection}

After anesthesia, transgenic or WT mice $(n=3-5)$ received $10 \mu$ intranasally $(5 \mu$ l each nostril $)$ of $1 \times$ phosphate buffer saline (PBS), LPS (10 $\mu \mathrm{g}$ in $1 \times \mathrm{PBS})$, dPG nanoparticles $(1 \mu \mathrm{M}$ in $1 \times \mathrm{PBS})$, and dPGS nanoparticles (1, $30 \mu \mathrm{M}$ in $1 \times \mathrm{PBS})$. The group of transgenic mice was then longitudinally imaged using in vivo bioluminescence.

\section{In vivo bioluminescent imaging}

In vivo imaging proceeded as previously described [30,36]. In short, an IVIS ${ }^{\circledR} 200$ Imaging System (PerkinElmer, MA, USA) was used to acquire bioluminescence images. GFAP-fluc mice or TLR2-luc-GFP mice were given intraperitoneal (ip.) injections of D-luciferine ( $150 \mathrm{mg} / \mathrm{kg}$, PerkinElmer) 20 or $25 \mathrm{~min}$, respectively before imaging. The grayscale photomicrographs were overlaid with a RGB gradient to indicate light intensity and quantification in the area of interest were normalized to photons per second per centimeter squared per steradian.

\section{Tissue collection \& immunofluorescence}

Twenty-four hours after intranasal administration, wild-type mice were anesthetized and transcardially perfused with PBS followed by $4 \%$ paraformaldehyde (PFA) solution. Tissue samples were extracted and kept in 4\% PFA overnight and transferred to phosphate-buffered $20 \%$ sucrose for cryopreservation. Coronal sections $(25 \mu \mathrm{m})$ of the olfactory bulb were generated and stored at $-20^{\circ} \mathrm{C}$. Immunohistochemistry was performed as previously described [32,35]. The primary antibodies are the following: 1:500 rabbit polyclonal anti-Ibal (microglia marker, Wako), 1:1000 mouse monoclonal GFAP (astrocyte marker, Cell Signaling) and 1:100 goat anti-LCN2 (R\&D Systems, ON, CA). Corresponding AlexaFluor secondary antibodies have been used at 1:500 dilution. Photomicrographs were acquired using a Zeiss LSM 700 Confocal microscope or Zeiss Axio Imager.

\section{Primary cortical dissociated cultures}

Cortices from P0-P3 C57BL6 mice were extracted and immersed in ice-cold Hank's Balanced Salt Solution. The tissue was dissociated mechanically by triturating through a glass pipette with a fire-polished bore. Dissociated cells were resuspended in culturing media containing Dulbecco's Modified Eagle's Medium (DMEM) $+10 \%$ fetal bovine serum $+1 \%$ penicillin-streptomycin and seeded onto Poly-D-Lysine coated coverslips or T75-flasks and cultured in a $37^{\circ} \mathrm{C}, 5 \% \mathrm{CO}_{2}$ incubator until confluence (7-12 days). Microglia were isolated by shaking a confluent culture seeded into a T75-flask in a $37^{\circ} \mathrm{C}$ shaker-incubator (G24 Environmental Incubator Shaker, New Brunswick Scientific, Edison, NJ, USA) at 200 r.p.m. for 2 h. Microglia were collected in the media and reseeded at 300,000 microglia/well into 24-well plates.

\section{Cell treatments}

Mixed glial cultures were treated with dPG and dPGS (synthesized as previously described) [37], or LPS (Escherichia coli, O111:B4, Sigma-Aldrich, ON, CA). TLR4 was inhibited using $10 \mu \mathrm{M}$ CLI-095 (Invitrogen) for $1 \mathrm{~h}$ prior to treatment with dPG or LPS. NF- $\kappa$ B was inhibited using $200 \mu \mathrm{M}$ caffeic acid phenethyl ester (CAPE; Millipore, ON, CA) for $1 \mathrm{~h}$ prior to LPS treatment. Acetylsalicylic acid (A5376-1006, Sigma-Aldrich) and N-acetyl-L-cysteine 
(A7250-256, Sigma-Aldrich) were administered as pretreatment for $3 \mathrm{~h}$ prior to LPS stimulation. To investigate the indirect effects of microglial secreted factors, primary microglia cultures were treated with LPS $\pm \mathrm{dPG}$ for $24 \mathrm{~h}$ prior to media collection and treatment of mixed glial cultures with microglia collected media (MCM) for $8 \mathrm{~h}$. To eliminate the effect of remnant LPS in the MCM, $10 \mu \mathrm{M}$ CLI-095 (TLR4 inhibitor) was added to MCM prior to administration of MCM to mixed glial cultures. In addition, $10 \mu \mathrm{M}$ CLI-095 pretreatment for $1 \mathrm{~h}$ was administered to mixed glial cultures prior to MCM treatment. To eliminate microglia in mixed cortical cultures, $15 \mu \mathrm{M}$ PLX5622 was added for 3 days prior to treatment which reduced microglia population to $<1 \%$ of the total cell population.

\section{Immunocytochemistry}

After treatment, cells were immersed in 4\% PFA for $10 \mathrm{~min}$ to fix and then blocked/permeabilized in PBS $+0.3 \%$ Triton-X100 $+0.5 \%$ BSA for $1 \mathrm{~h}$ at room temperature (RT). After blocking, cells were incubated in primary antibody diluted into a solution of PBS $+0.3 \%$ Triton-X100 for $1 \mathrm{~h}$ at RT. Primary antibodies used: goat antiLCN2 (R\&D Systems, 1:40), rabbit anti-GFAP (Abcam, ON, CA, 1:1000), and rabbit anti-Iba1 (Thermofisher, Mississauga, ON, CA, 1:500). After washing, cells were labeled with appropriate secondary antibodies diluted in PBS for $1 \mathrm{~h}$ at RT. Secondary antibodies used: AlexaFluor-488 donkey anti-goat (Thermo Fisher Scientific, 1:500) and AlexaFluor-647 donkey anti-rabbit (Thermo Fisher Scientific 1:500). Nuclei were labeled by immersing cells in $10 \mu \mathrm{M}$ Hoechst 33342 for $10 \mathrm{~min}$ at RT. Coverslips were mounted onto microscope slides using Aqua-Poly/Mount (Polysciences, Inc., PA, USA) and images were acquired using a fluorescence microscope (Leica DMI4000B, Leica Microsystems, ON, CA). Intracellular fluorescence was measured within the cell outline, and background was subtracted.

\section{SPR-based binding assays}

Recombinant proteins mouse LCN2 and mouse IL6 were purchased from Biotechne and Peprotech, respectively. Binding studies were performed on a Biacore X100 instrument at $25^{\circ} \mathrm{C}$, using HBS-EP+ $(10 \mathrm{mM} \mathrm{HEPES}, \mathrm{pH} 7.4$, $150 \mathrm{mM} \mathrm{NaCl}, 0.05 \%$ Surfactant P20) for IL6 binding measurements. Furthermore, sodium phosphate buffer (10 mM Na-PO4 pH 7.0) for LCN2 was investigated. Biotinylated dPGS was immobilized on a streptavidin sensor chip (GE Healthcare, IL, USA, final response 120.3 RU), whereas the reference lane was immobilized with biotinylated dPG-OH (138.9 RU). Binding affinities were quantified by kinetic titration series. Five sample dilutions were serially injected at a flow rate of $30 \mu \mathrm{L} /$ minute. Each sample was measured in duplicates. Each cycle bore on a 180 seconds period of sample contact time (association phase) followed by a $600 \mathrm{~s}$ dissociation phase. The final regeneration was achieved with $5 \mathrm{M} \mathrm{NaCl}$ for $90 \mathrm{~s}$, followed by $50 \mathrm{mM} \mathrm{NaOH}$ for $30 \mathrm{~s}$. The determination of $\mathrm{KD}$ values was performed with response unit (RU) data points taken at $4 \mathrm{~s}$ before injection stop using the built-in software plus package. Corresponding binding isotherms were plotted.

\section{Statistical analysis}

Statistical significance was assessed using one way ANOVAs with post hoc test and Student's t-test. A significance threshold of $\mathrm{p}<0.05$ was used. Graphs are presented as the mean values with error bars representing the SEM.

\section{Results}

We first addressed the question if LPS and dendritic polyglycerols with $\mathrm{OH}(\mathrm{dPG})$ and sulfate terminal groups (dPGS) activate microglia and astrocytes in vivo and if this activation leads to an increase in LCN2 protein abundance in these cells. We used transgenic mice expressing luciferase driven by TLR2 or GFAP promoter [33]. Longitudinal assessment of microglia activation/innate immune response in transgenic mice receiving intranasal LPS and LPS + dPG and in a lesser extent dPG alone, show an induction of the TLR2 signal starting at $6 \mathrm{~h}$ and reaching a maximum at $24 \mathrm{~h}$ after initial LPS challenge (Figure 1). Importantly, our previous study clearly demonstrated that induction of TLR2 signaling observed by bioluminescence imaging can be used as a surrogate marker of microglial activation in response to LPS challenge [30]. Indeed, immunohistochemical analysis of mice brain sections (olfactory bulb region) revealed that an increase in TLR2 signal was associated with changes in microglia morphology and increase in immunoreactivity for ionized calcium binding adaptor molecule 1 (Iba1) in LPS and LPS + dPG treated mice when compared with control, PBS treated animals (Figure 1O-Q). dPG administration alone induced only modest changes in Iba1 when compared with control sections. 


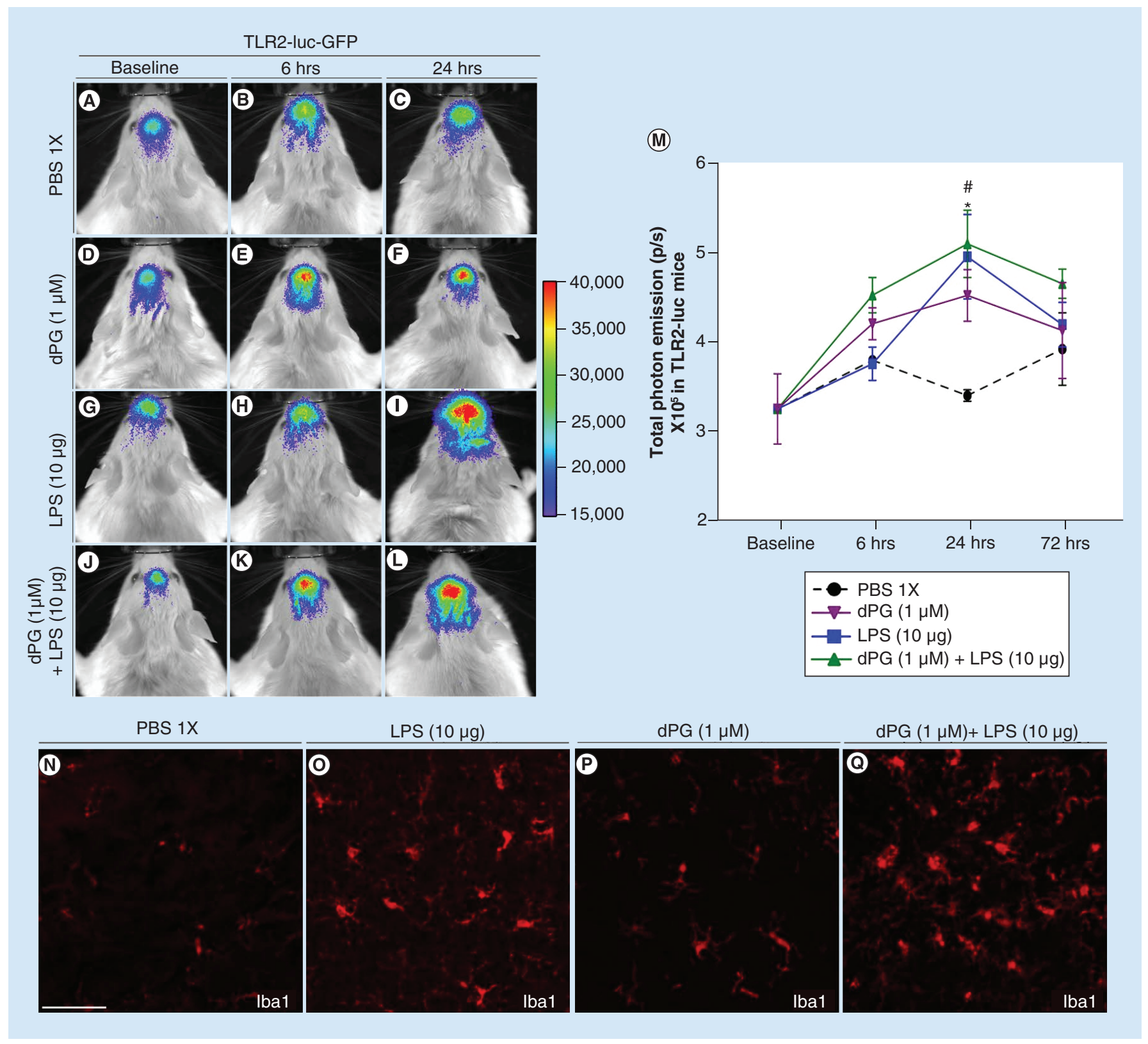

Figure 1. Live imaging of innate immune response and microglia activation in TLR2-luc-GFP mice. dPG: Dendritic polyglycerol; LPS: Lipopolysaccharide; PBS: Phosphate-buffered saline.

Contrary to innate immune response and microglia activation observed in TLR2 reporter mice, in vivo imaging of the GFAP signal revealed that astrocytes were significantly activated only with LPS. Intranasal delivery of LPS + dPG and/or dPG alone did not have significant effect on the GFAP signal induction and/or astrocyte activation (Figure 2). Astrocyte morphology was comparable after both treatments and did not differ from those animals with intranasally administered PBS (Figure $2 \mathrm{~N} \& \mathrm{Q}$ ).

\section{Upregulation of LCN2 in astrocytes in vivo \& in mixed mouse cortical cultures}

Astrocytes produce LCN2 in response to insult [16], but its role depends on the type of stimuli, intensity and duration. We recapitulated LCN2 production in astrocytes in response to LPS and LPS + dPG stimulation in primary mouse cortical cultures. Dual labeling of astrocytes with antibodies against GFAP and LCN2 revealed an increased LCN2 protein abundance in astrocytes but not in Iba1-immunopositive microglia (Figure 3A \& Supplementary Figure 


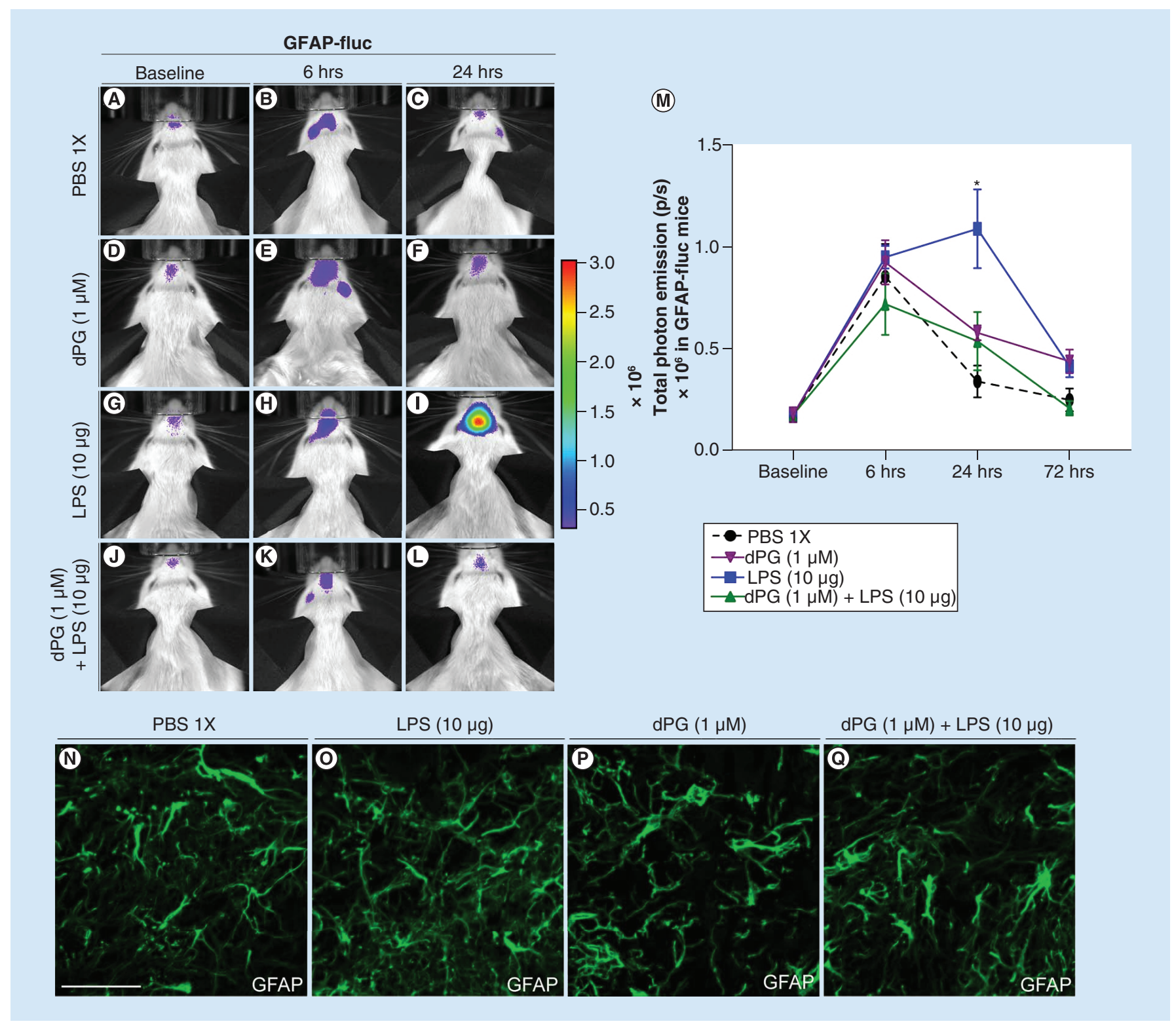

Figure 2. Live imaging of astrocyte activation in GFAP-fluc mice.

dPG: Dendritic polyglycerol; LPS: Lipopolysaccharide; PBS: Phosphate-buffered saline.

1).

Concentration and time-dependent LCN2 abundance in dissociated mouse cortical cultures containing astrocytes, microglia and neurons (treated with dPG and LPS) show significant LCN2 protein content in astrocytes after 24-h post-LPS administration with or without dPG suggesting that the main activation derives from LPS and not from dPG (Figure 3A-D). These findings were in line with our results from in vivo studies (Figures $1 \& 2$ ).

Although $10 \%$ of cells in mixed cortical cultures are microglia, they are not the main source for LCN2 production based on the barely detectable signal from only about $13 \%$ of microglia with somewhat upregulated LCN2. This was confirmed with enriched microglia cultures treated with LPS or dPG for $24 \mathrm{~h}$. In contrast, about $90 \%$ of astrocytes in mixed cortical cultures showed increased LCN2 protein abundance (Figure 3A). 

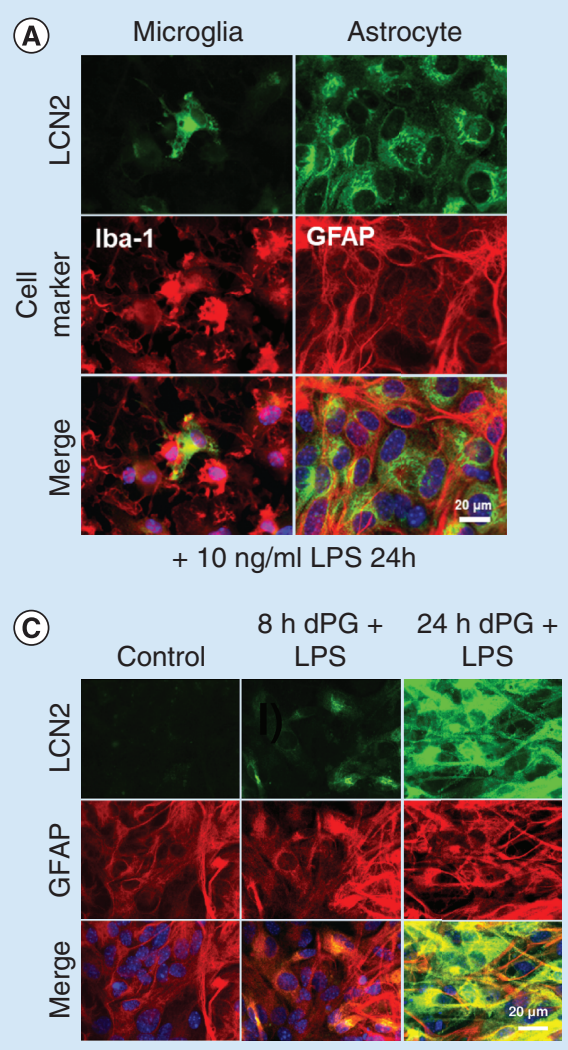

(B)

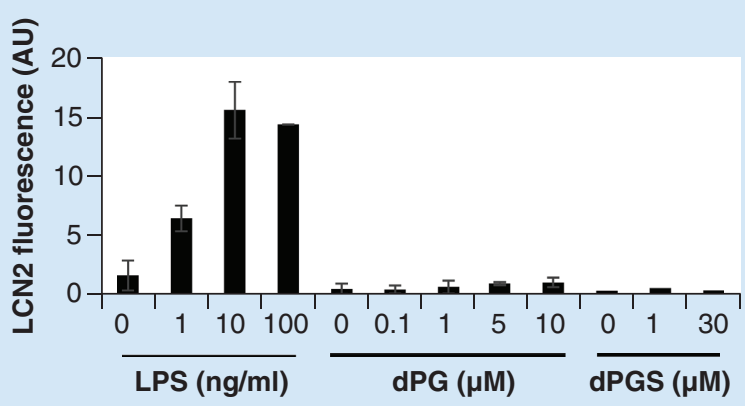

(D)

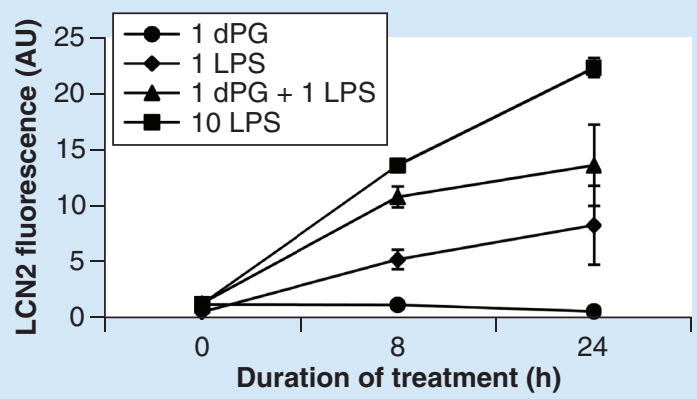

Figure 3. Astrocytic LCN2 production in response to dose and time-dependent lipopolysaccharide, dendritic polyglycerol, and sulfated dendritic polyglycerol treatment.

dPG: Dendritic polyglycerol; LPS: Lipopolysaccharide.

\section{dPGS significantly reduce activation of microglia in vivo in a concentration \& time-dependent manner}

In stark contrast to LPS, dPGS reduced activation of microglia. Real time bioluminescent imaging of TLR2-LucGFP mice showed significant reduction of total photon emission in mice exposed to LPS and co-treated with dPGS $(30 \mu \mathrm{M})$ but not with dPGS in low concentration $(1 \mu \mathrm{M})$. Intranasal delivery of dPGS alone $(1$ and $30 \mu \mathrm{M})$ did not induce increase in the TLR2 signals. In keeping with in vivo imaging results, immunofluorescence analysis revealed that co-treatment with dPGS $(30 \mu \mathrm{M})$ markedly attenuated increase in Iba1 immunoreactivity. Next, delivery of dPGS alone did not induce changes in morphology of microglial cells nor Iba1 immunoreactivity. Taken together, dendritic polyglycerols with OH (dPG) could not suppress the LPS-induced effects on microglia. In contrast, dendritic polyglycerols with sulfate terminal groups (dPGS) exert anti-inflammatory properties, in the context of innate immune challenge, and markedly attenuated LPS-mediated effects on microglia (Figure 4).

In agreement with these in vivo results, studies conducted in primary dissociated mixed cortical cultures showed that dPGS $(30 \mu \mathrm{M})$ treatment reduce $>50 \%$ LCN2 abundance in activated astrocytes whereas $1 \mu \mathrm{M}$ dPGS only marginally reduced LCN2 expression (Figure 5A \& B). Recent literature has suggested that microglia may play a crucial role in the activation and inflammatory response of astrocytes by secreting pro-inflammatory cytokines that can activate surrounding astrocytes [28].

To show that the main stimulus deriving from microglia exposed to LPS is a soluble factor in the MCM, we treated microglia with CSF-1 pharmacological inhibitor PLX5622. This inhibitor was previously used for microglia ablation [38]. Indeed, our results show that mixed cortical cultures treated with PLX5662 for 3 days do not release factors that activate astrocytes and enhance LCN2 (Figure 6). In contrast, MCM from LPS-treated microglia significantly enhanced LCN2 abundance in astrocytes (Figure 6) [28]. 


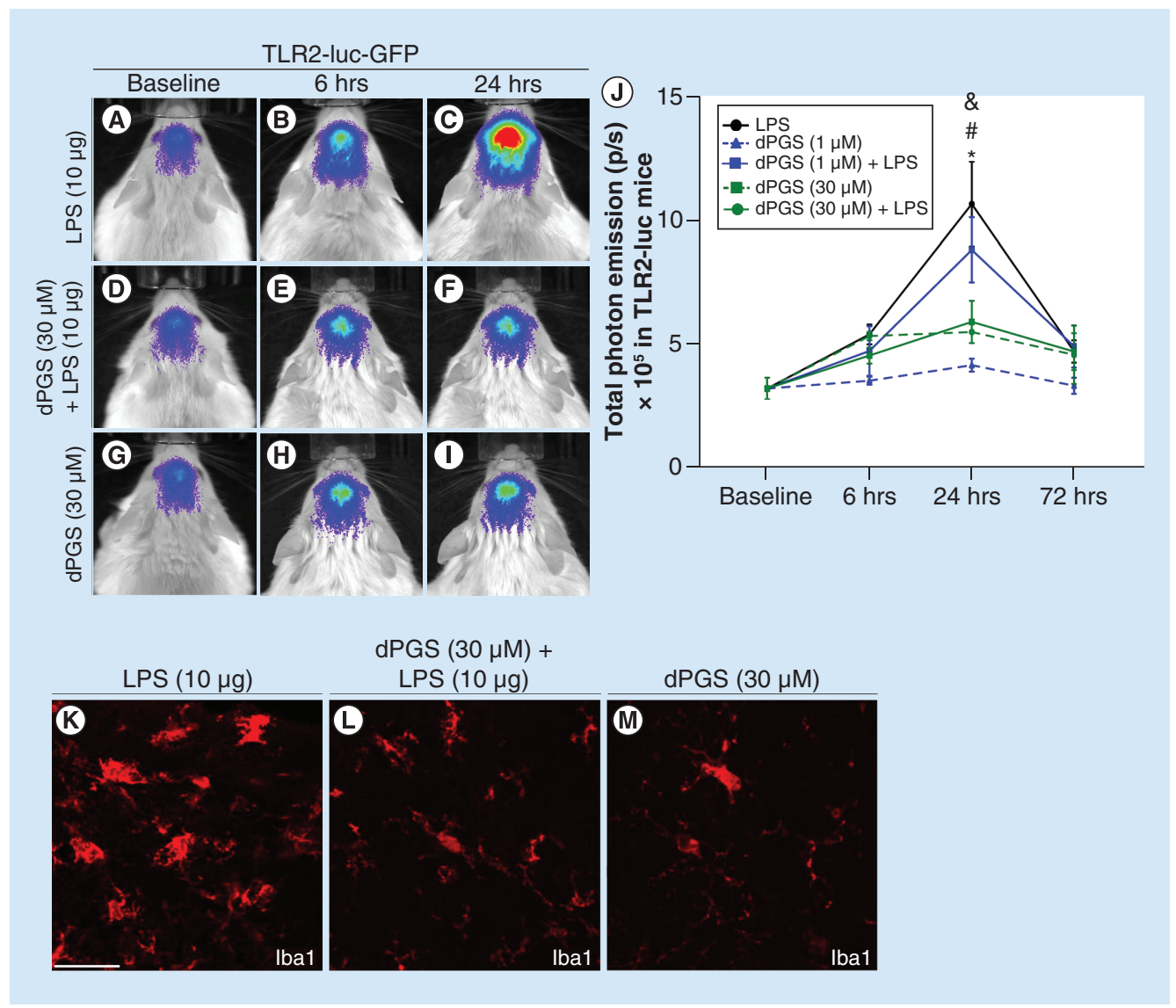

Figure 4. Sulfated dendritic polyglycerol reduces microglial activation in TLR2-luc-GFP mice. dPGS: Sulfated dendritic polyglycerol; LPS: Lipopolysaccharide.

\section{Mechanisms of LCN2 regulation in astrocytes implicate TLR4-NFkB axis \& IL6}

Considering that LPS and LPS + dPG indirectly induced LCN2 in astrocyte through microglia-secreted soluble factors, we investigated if the TLR4-NFkB axis is implicated in regulation of LCN2 in astrocytes. LPS and LPS + dPG induced NF- $\mathrm{KB}$ nuclear translocation in microglia at a maximum $1 \mathrm{~h}$ after treatment. The translocation peak in similarly treated astrocytes was delayed (maximum after $4 \mathrm{~h}$ treatment). Blocking TLR4 with pharmacological inhibitor CLI-095 significantly reduced LCN2 protein in activated astrocytes (Figure 7A \& B). Similarly, treatment of the enriched astrocytic cultures with CAPE, an inhibitor of NFkB translocation from the cytosol to the nucleus, significantly reduced LCN2 abundance in LPS-stimulated cultures (Figure 7C \& D).

The canonical pathway of LPS signaling requires TLR4 activation in order to induce a pro-inflammatory response in its target cells. Based on the previously reported LPS mechanisms of action, we hypothesized that this pro-inflammagen will upregulate cytokine IL-6 in microglia which will be released and stimulate astrocytes. Our earlier studies showed that IL-6 is upregulated in microglia and in organotypic mouse brain slices and that dPGS treatment can significantly reduce IL-6 upregulation [2]. IL-6 binds to sulfated glucosaminoglycans (GAGs) [39]. In a competitive ELISA approach, an $\mathrm{IC}_{50}$ of $200 \mathrm{nM}$ was reported. Human IL-6 binds to immobilized heparin-BSA complexes and it was displaced by soluble heparin [40]. In the present study, we used SPR and determined a $K_{D}$ of $488 \mathrm{nM}$ (Figure 7E \& F). We assume that dPGS act as a soluble scavenger of IL-6 delivered by activated microglia and thus reduce astrocyte activation. In addition, binding of LCN2 to surface immobilized dPGS was also confirmed (Supplementary Figure 2).

\section{$\mathrm{N}$-acetylcysteine mitigates induction of LCN2 in astrocytes}

Previous studies using LPS in neural cultures and animal models suggested that ROS play a role in releasing cytokines and alarmins from microglia $[41,42]$. To determine if ROS was an intermediate step in LPS \pm dPG-induced LCN2 

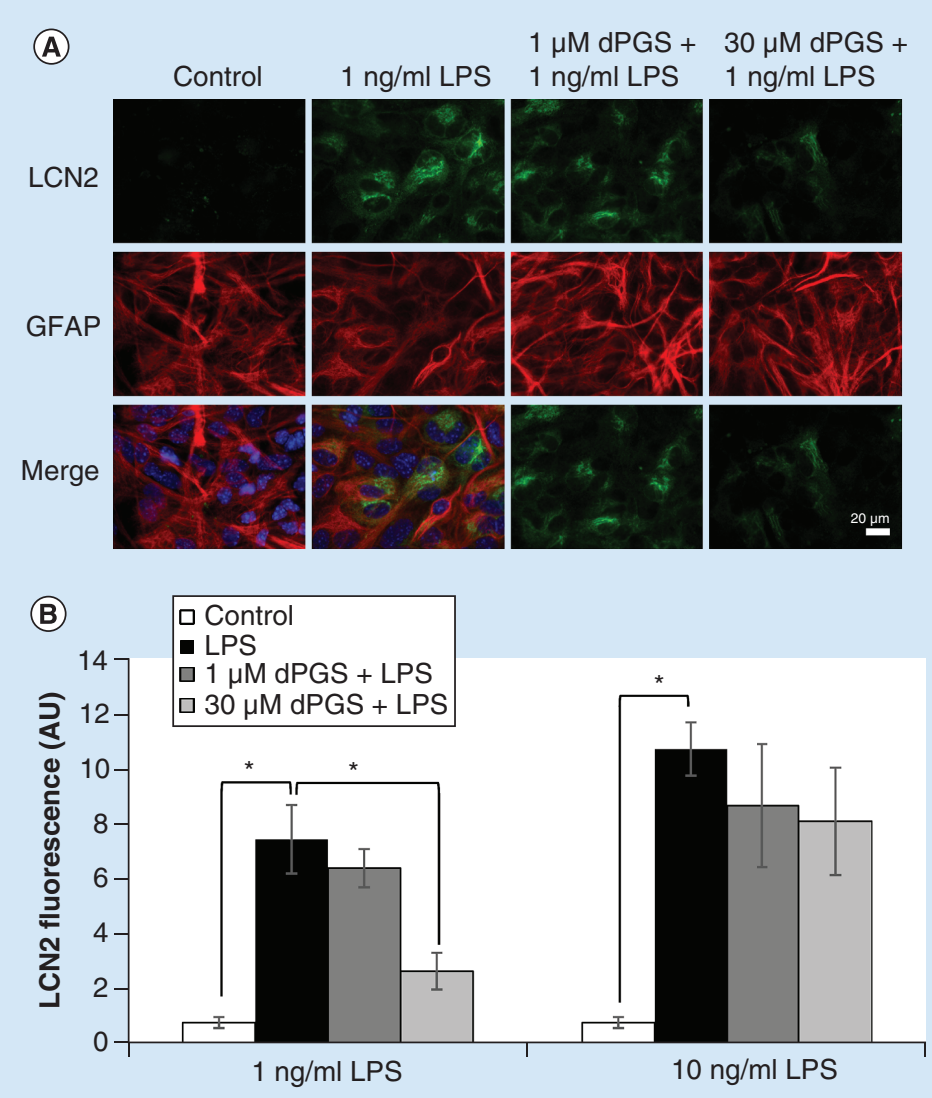

Figure 5. Sulfated dendritic polyglycerol reduces astrocytic LCN2 production in mixed cortical cultures. dPGS: Sulfated dendritic polyglycerol; LPS: Lipopolysaccharide.

upregulation, we used N-acetylcysteine (NAC) to reduce ROS concentrations in mixed neural cultures (Figure 7G $\& \mathrm{H}$ ). NAC is commonly employed ROS scavenger, anti-inflammatory agent and anti-oxidant [43]. NAC treatment $3 \mathrm{~h}$ prior to the LPS $\pm \mathrm{dPG}$ revealed that ROS was implicated in LCN2 regulation. NAC significantly reduced the abundance of LCN2 in astrocytes (Figure 7G \& H) when LPS \pm dPG was added to mixed cortical cultures for $8 \mathrm{~h}$. Similarly, when NAC was added to primary mouse microglia cultures, prior to the addition of LPS, LPS+ dPG or MCM; NAC presence in the media significantly reduced LCN2 concentrations in astrocytes (Figure 7G $\& \mathrm{H})$ pointing toward the role(s) of ROS in the mechanism of LCN2 regulation in astrocytes.

\section{Discussion}

Results from the presented studies suggest that dendritic polyglycerols with sulfate groups (dPGS) regulate microglia activity and LCN2 protein abundance in astrocytes. LPS-stimulated microglia or media from such cells are powerful inducers of LCN2 synthesis in astrocytes. LPS causes significant enhancement of LCN2 abundance in astrocytes. Mechanisms implicated in LCN2 regulatory processes in astrocytes include the participation of TLR4-NFkB axis, IL6 and ROS. So far, only a few studies with dPGS have been performed in primary neuroglia, and none in vivo, in transgenic animals.

Dendritic polyglycerols have been tested in various in vitro and in vivo models [2,3,13]. dPGS were proven to exert remarkable anti-inflammatory activity in models of peripheral inflammation such as dermatitis and myositis [3,44]. Targeting of the cell adhesion molecules L- and P-selectin, as well as proteins of the complement cascade dampened the inflammatory response. Previous studies by our group show that LCN2 mRNA is not translated into protein in microglia, but LCN2 protein is abundant in astrocytes upon LPS exposure [25]. Upregulation of LCN2 has been shown in different tissues under stressful conditions and exposure to pathogens [45]. Results for LCN2 regulation in astrocytes in the presence of nanomaterials (e.g., dendritic polyglycerols) have not been reported. Considering that 


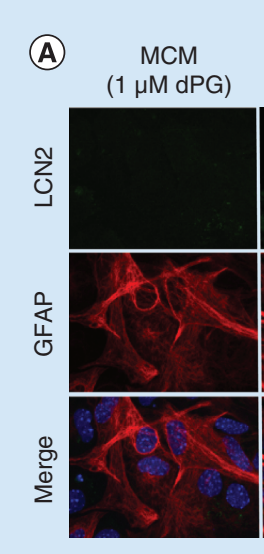

(C)

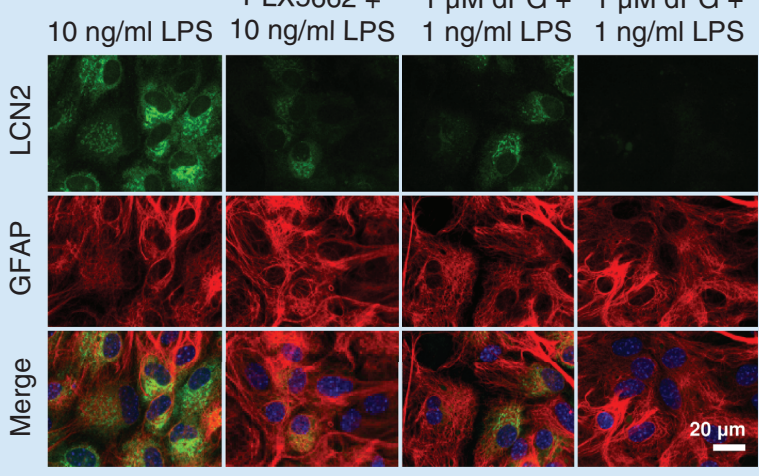

(B)

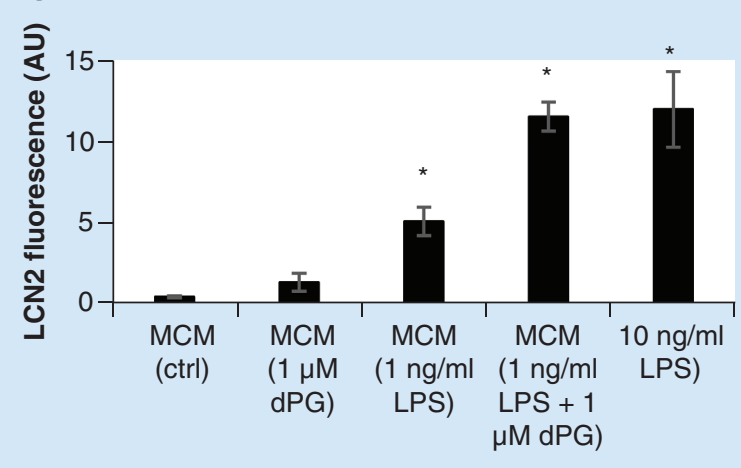

(D)

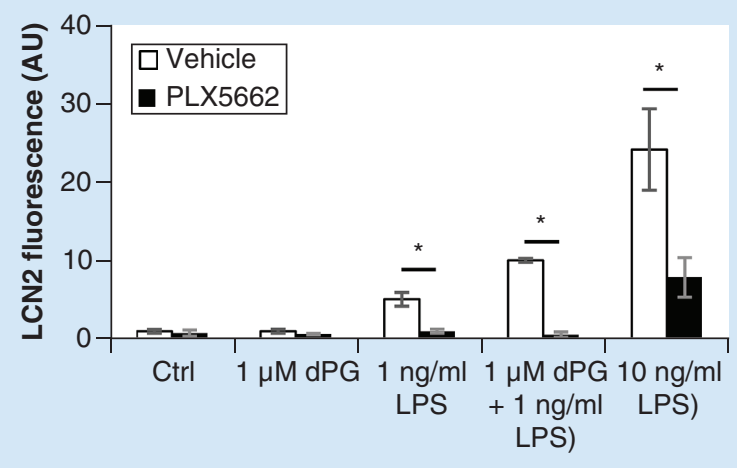

Figure 6. Astrocytic LCN2 production is dependent on microglia-secreted factors.

dPGS: Sulfated dendritic polyglycerol; LPS: Lipopolysaccharide; MCM: Microglia collected media.

LPS is a powerful LCN2 inducer and that dendritic polyglycerols have forged their way toward clinical applications, we performed experiments to answer the following questions: Is neuroglia activation by dendritic polyglycerols temporally and globally similar to that induced by LPS; are microglia necessary for astrocyte activation and LCN2 production; and what are the key steps leading to LCN2 upregulation? Results from our current studies suggest that dPGS and $\mathrm{dPG}$ do not markedly activate microglia in vivo in transgenic mice expressing luciferase under the control of neuroglia promoter TLR2. mRNA and protein profiles in activated microglia indicate divergent networks whereby high content of mRNA are not translated into proteins in LPS-stimulated microglia. LCN2 mRNA in LPS-activated microglia is not translated into LCN2 protein because of powerful microglial translational suppressor, RNA-binding protein SRSF3. Whether the mechanism suppressing LCN2 mRNA translation is still active when microglia are exposed to nanostructured materials is an intriguing question that merits further investigation.

LCN2 was mainly produced by activated astrocytes despite a significant upregulation of LCN2 mRNA in microglia [25]. Dual labeling of LCN2 and astrocytic marker GFAP clearly showed marked increase of LCN2 in astrocytes. To complement these findings, we also pharmacologically blocked CSF1R with PLX5622. CSF1R is necessary for microglia proliferation and survival [46,47]. Inhibition of CSF1R with PLX5622 significantly and selectively reduce microglia [38]. In this study, PLX5622 significantly reduced the number of microglia within a week to a barely detectable level; this resulted with a significant LCN2 protein reduction in astrocyte in the mixed cortical cultures. Taken together, our studies suggest that LPS $\pm \mathrm{dPG}$-activated microglia are powerful regulators of astrocytic LCN2. Binding of secreted LCN2 will probably influence the local iron metabolism. This is of interest, because iron accumulation has been observed in CNS disorders like Parkinson's disease and Alzheimer's disease $[48,49]$.

Mechanisms for LCN2 regulation in astrocytes implicate TLR4-NFkB axis. LPS concentration used in our studies did not lead to significant cell death, but it can impair mitochondrial functions [50]. Even transient mitochondrial 


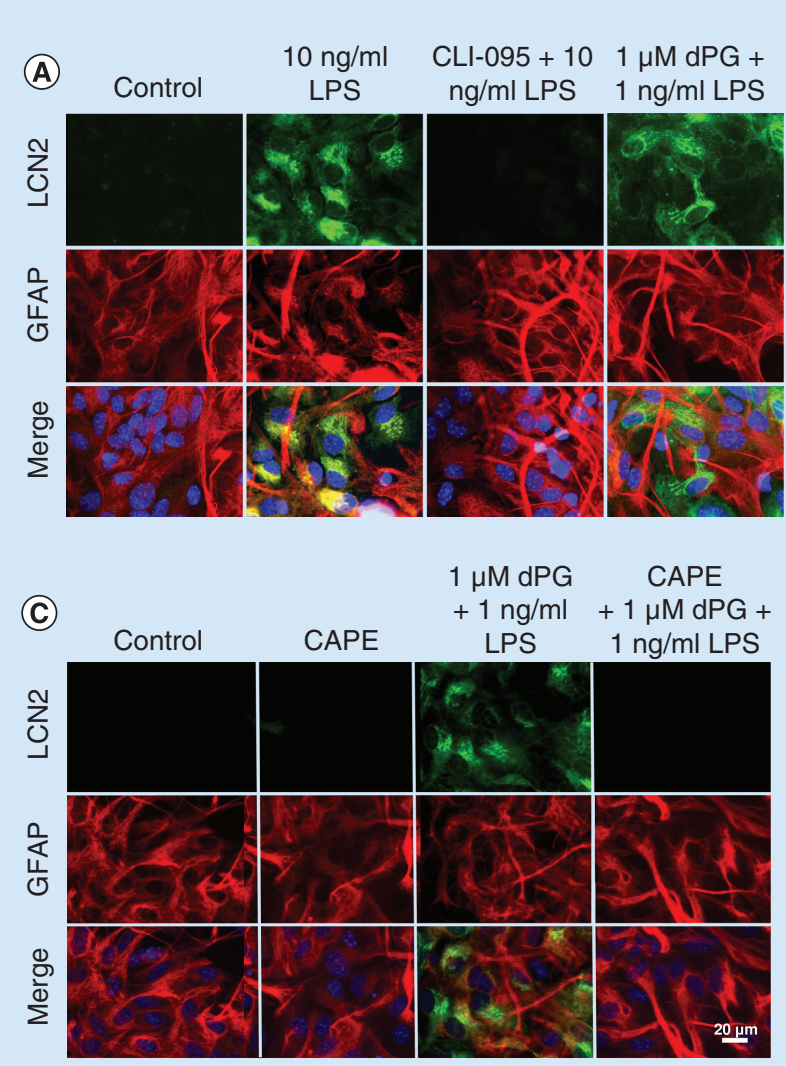

$\mathrm{CLI}-095+$

$1 \mu \mathrm{M} \mathrm{dPG}+$ $1 \mathrm{ng} / \mathrm{ml}$ LPS

(B)
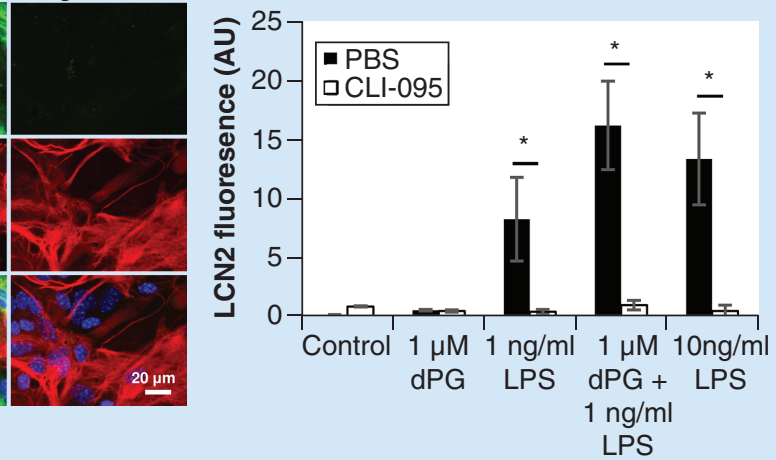

(E)

(F)

(D)
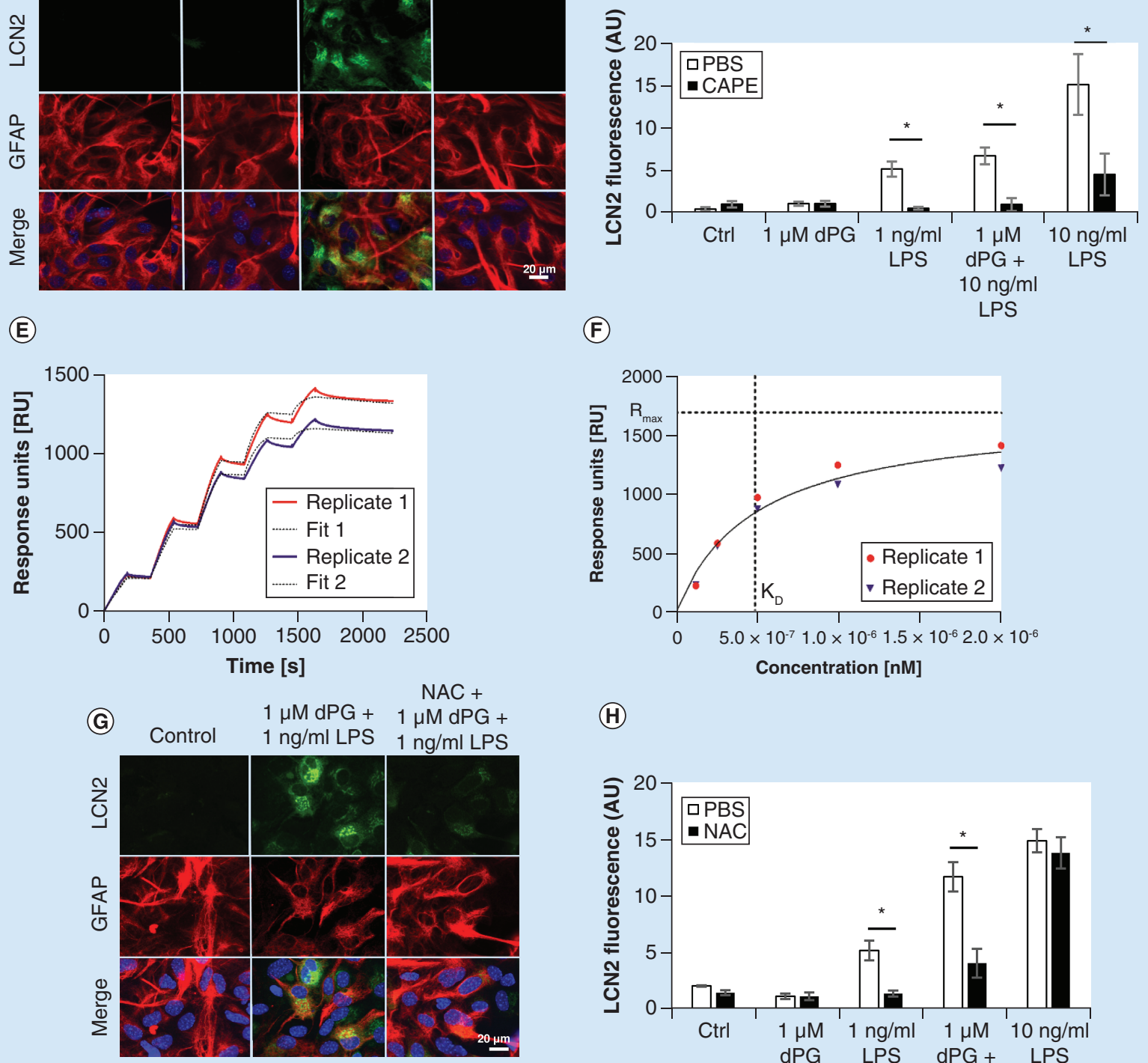

(H)

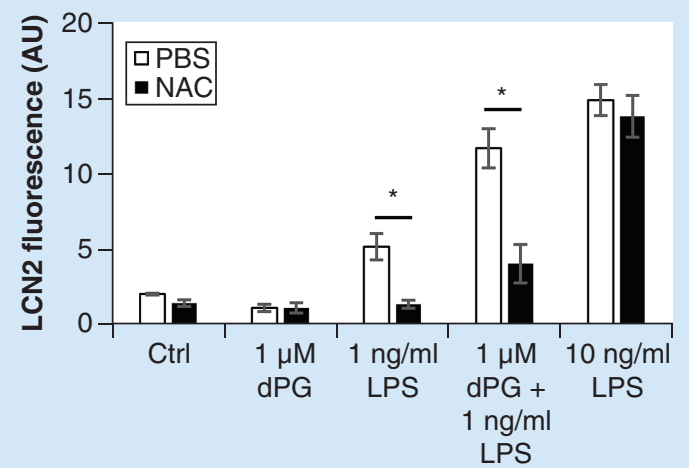

Figure 7. Pathways implicated in LCN2 production and sulfated dendritic polyglycerol activity. CAPE: Caffeic acid phenethyl ester; dPG: Dendritic polyglycerol; LPS: Lipopolysaccharide; PBS: Phosphate-buffered saline; NAC: $\mathrm{N}$-acetylcysteine. 
dysfunction and oxidative stress can lead to oxidation of mitochondrial components (e.g., mitochondrial mtDNA, cardiolipin) and the release of mitochondria-derived damage-associated molecular patterns (DAMPS) [51]. DAMPs are damage signaling molecules which activate microglia by binding to TLR receptors and contribute to their functional impairment [52]. As a consequence of the binding of DAMPs to TLR and other receptors expressed on immune cells including neuroglia, activated cells release largely pro-inflammatory cytokines [53,54]. In addition, mitochondrial DAMPs, mtDNA, TFAM, cardiolipin released from injured mitochondria induce activation of inflammasomes and the release of cytokines. For example, IL-1 $\beta$ and IL- 6 act in an autocrine and paracrine manner to form a vicious circle with inflammasome-mediated pathways, reinforcing the neuroglia activating (inflammatory) response [55]. dPGS can block (at least in part) this vicious cycle by binding to IL-6.

Our results with TLR4 inhibitor CLI-095 suggest that signaling via TLR4 plays a substantial role in LCN2 regulation. CLI-095 inhibited LCN2 upregulation in astrocytes treated with LPS, which suggests a TLR4-dependent mechanism of action. LCN2 upregulation in astrocytes is largely induced by microglia through an indirect mechanism considering that these receptors are mainly present on the surface of microglia. A small proportion of TLR4-positive astrocytes was found in juvenile mice and in human astrocytes [56,57]. However, most studies show TLR4 in mice microglia implicating these receptors in microglia stimulation and subsequent cytokine release, for example, IL-1 and IL-6. Our studies show that sulfated polyglycerol dendrimers (dPGS) bind IL-6 which can explain attenuation of LCN2 synthesis and abundance of LCN2 in astrocytes.

Excessive ROS in conjunction with mitochondrial impairment was mitigated by NAC with treatment of mixed cortical cultures or enriched microglia prior to dPG treatment. NAC is an antioxidant, free radical scavenger, glutathione precursor and an inhibitor of NF- $\mathrm{KB}[58,59]$. Thus, pleiotropic NAC could lead to a reduction in oxidative stress and suppression of cytokine production by preventing NF-KB nuclear translocation. This mechanism was reported for LPS-stimulated glial cultures which secreted IL-1 $\beta$, TNF- $\alpha$, IL6 and increased nitric oxide synthase and translocation of NF- $\kappa B$ [60,61]. Our experiments using an inhibitor of NF- $\kappa$ B activation (CAPE) show that this transcription factor plays a critical role in LCN2 upregulation in astrocytes; CAPE significantly reduced LCN2 abundance in these neuroglial cells. A further detailed analysis on the iron-siderophore binding protein LCN2 and its complex with dPGS is of high interest. It has been described that the expression of hepcidin, a major regulator of iron homeostasis, is inhibited by the polysulfate heparin [62]. Hepcidin expression itself is triggered by the BMP/SMAD signaling pathway and the inflammatory IL-6/STAT3 axis. Regarding the iron deposition in CNS diseases the role of dPGS is unknown and merits further investigations.

\section{Conclusion}

We show the immunomodulatory effect of dendritic polyglycerols terminating in hydroxyl or sulfate groups (Figure 8). In the absence of pro-inflammatory stimulus LPS, neither dPG nor dPGS treatment induced LCN2 biosynthesis. When combined with LPS-stimulation, dPGS treatment reduced the production LCN2 protein in mixed glial cultures. Although LPS stimulated both astrocytes and microglia in vivo, LCN2 production took place mainly in astrocytes. Furthermore, microglia activation was necessary to trigger astrocyte LCN2 production which involved the release of cytokines, including IL-6, from microglia.

Binding of IL- 6 to dPGS reduced the simulation of astrocytes and LCN2 abundance. The mechanism of LCN2

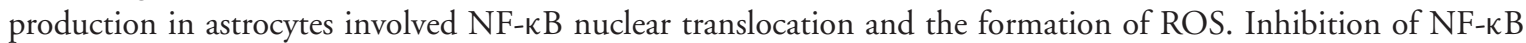
nuclear translocation using caffeic acid phenethyl ester or neutralizing ROS using $\mathrm{N}$-acetylcysteine dramatically lowered LCN2 abundance in astrocyte stimulated with LPS or proinflammatory cytokines secreted by activated microglia. Sulfated dendritic polyglycerols significantly decreased the extent of microglia activation in vivo and may be useful nanostructures as modulators in neuroinflammation.

\section{Future perspective}

Dendritic polyglycerols (dPGS) have emerged as effective nanotechnological modulators of diverse cellular functions. Previous studies have shown that dPGS can prevent dendritic spine loss in organotypic cultures exposed to LPS or amyloid $\beta$ oligomers. The present study shows that dPGS administered noninvasively in transgenic mouse model of neuroinflammation significantly reduce microglia activation and production of LCN2 in astrocytes. Given that dPGS are highly biocompatible, can act as tunable drug carriers, and possess intrinsic anti-inflammatory properties, dPGS are strong candidates for testing in nonhuman primates and eventually in humans. dPGS could be of particular relevance in treating neurological disorders with or without associated neuroinflammation. 


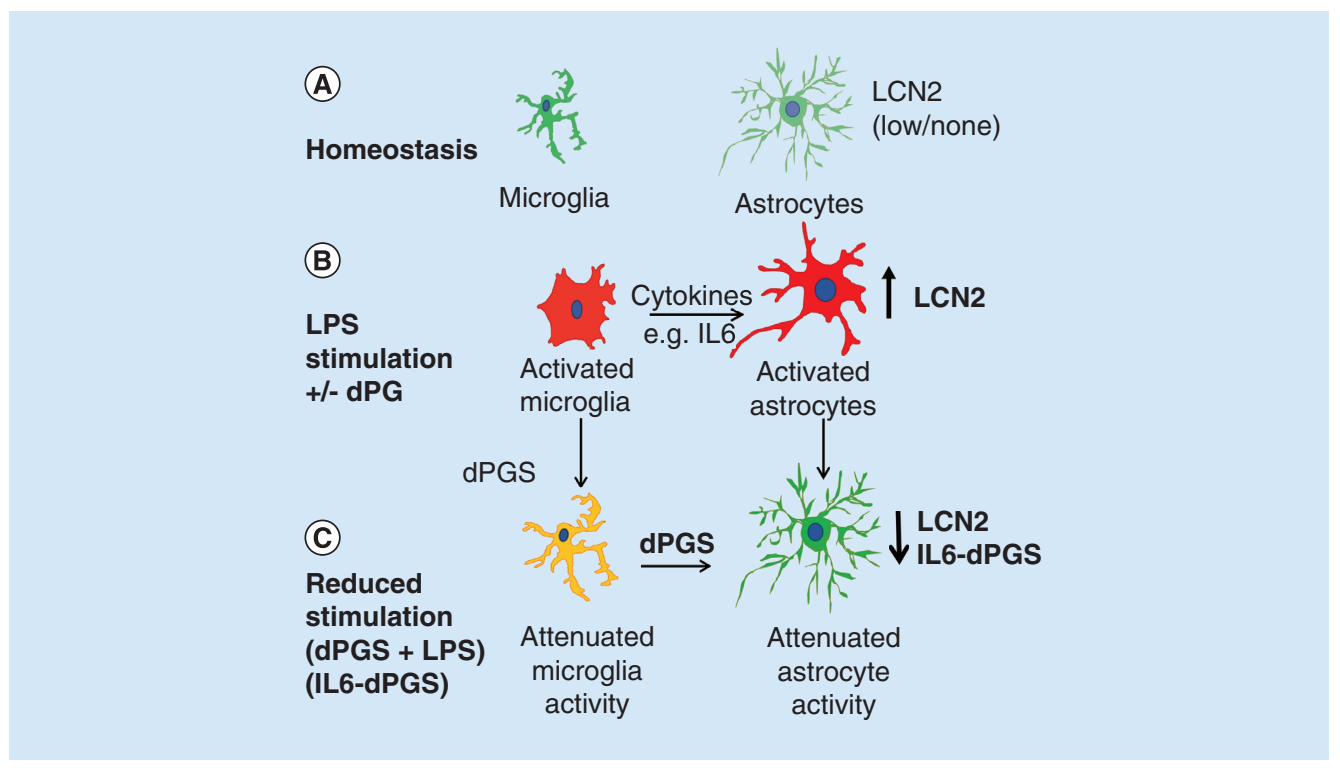

Figure 8. Schematic overview of microglia-astrocyte crosstalk.

\section{Executive summary}

- Dendritic polyglycerols (dPGS) do not activate microglia or astrocytes in primary neural cultures or in vivo.

- dPGS do not upregulate LCN2 in astrocytes.

- dPGS reduce LPS-stimulated microglia activation in vivo.

- $\mathrm{dPGS}$ reduce LPS-stimulated astrocytic LCN2 production.

- LCN2 production by astrocytes requires stimulated microglia.

- dPGS bind to IL-6, which interrupts microglia-astrocyte crosstalk.

- Astrocytic LCN2 abundance is significantly reduced by inhibition of NF-kB activation and reactive oxygen species elimination.

\section{Author contributions}

M Lalancette-Hébert performed the in vivo biological experiments. J Ji and K Jabbour performed the in vitro biological experiments. J Dernedde and $\mathrm{K}$ Silberreis performed the surface plasmon resonance experiments. $\mathrm{R}$ Haag provided the dendrimers and participated in manuscript preparation. D Maysinger initiated the project and wrote the manuscript with help from all co-authors. D Maysinger, R Haag and J Kriz are the senior investigators in this project.

Financial \& competing interests disclosure

D Maysinger and J Kriz acknowledge financial support by the Canadian Institute of Health Research (CIHR: MOP-89995). D Maysinger also acknowledges the support of from the Natural Sciences and Engineering Research Council (NSERC: STPGP 41346811). J Dernedde and R Haag thank the collaborative research center SFB 765 by the Deutsche Forschungsgemeinschaft (DFG) for financial support. The authors have no other relevant affiliations or financial involvement with any organization or entity with a financial interest in or financial conflict with the subject matter or materials discussed in the manuscript. This includes employment, consultancies, honoraria, stock ownership or options, expert testimony, grants or patents received or pending, or royalties.

No writing assistance was utilized in the production of this manuscript.

\section{Ethical conduct of research}

All experimental procedures were approved by the Université Laval Animal Care Ethics Committee (protocol \#14-096-4), the Ethics Committee for Animal Research at McGill University (protocol \#2008-5521), and are in accordance with the Guide to the Care and Use of Experimental Animals of the Canadian Council on Animal Care. 
Open access

This work is licensed under the Creative Commons Attribution-NonCommercial-NoDerivatives 4.0 Unported License. To view a copy of this license, visit: http://creativecommons.org/licenses/by-nc-nd/4.0/

\section{References}

Papers of special interested have been noted as: • of interest; $\bullet \bullet$ of considerable interest

1. Hayder M, Fruchon S, Fournie JJ, Poupot M, Poupot R. Anti-inflammatory properties of dendrimers per se. Sci. World J. 11, 1367-1382 (2011).

2. Maysinger D, Gröger D, Lake A et al. Dendritic polyglycerol sulfate inhibits microglial activation and reduces hippocampal CA1 dendritic spine morphology deficits. Biomacromolecules 16(9), 3073-3082 (2015).

-• Highlights the effect of dPGS on reversing dendritic spine loss in organotypic cultures.

3. Dernedde J, Rausch A, Weinhart M et al. Dendritic polyglycerol sulfates as multivalent inhibitors of inflammation. 107(46), 19679-19684 (2010).

-• Highlights the interaction of dPGS with peripheral immune cells and selective inhibition on L- and P-selectins.

4. Tomalia DA, Fréchet JMJ. Discovery of dendrimers and dendritic polymers: a brief historical perspective. J. Polymer Sci. Part A 40(16), 2719-2728 (2002).

5. Tomalia DA, Naylor AM, Goddard Iii WA. Starburst dendrimers: molecular-level control of size, shape, surface chemistry, topology, and flexibility from atoms to macroscopic matter. Angewandte Chemie 29(2), 138-175 (1990).

6. Fruchon S, Poupot R. Pro-inflammatory versus anti-inflammatory effects of dendrimers: the two faces of immuno-modulatory nanoparticles. Nanomaterials 7(9), 251 (2017).

7. Pant K, Pufe J, Zarschler K et al. Surface charge and particle size determine the metabolic fate of dendritic polyglycerols. Nanoscale 9(25), 8723-8739 (2017).

8. Madaan K, Kumar S, Poonia N, Lather V, Pandita D. Dendrimers in drug delivery and targeting: drug-dendrimer interactions and toxicity issues. J. Pharm. Bioall. Sci. 6(3), 139-150 (2014).

9. Wolinsky JB, Grinstaff MW. Therapeutic and diagnostic applications of dendrimers for cancer treatment. Adv. Drug Deliv. Rev. 60(9), 1037-1055 (2008).

10. Shaunak S, Thomas S, Gianasi E et al. Polyvalent dendrimer glucosamine conjugates prevent scar tissue formation. Nat. Biotech. 22(8), 977-984 (2004).

11. Schneider T, Welker P, Licha K, Haag R, Schulze-Tanzil G. Influence of dendritic polyglycerol sulfates on knee osteoarthritis: an experimental study in the rat osteoarthritis model. BMC Musculoskelet. Discord. 16, 387 (2015).

12. Schneider T, Welker P, Haag R et al. Effects of dendritic polyglycerol sulfate on articular chondrocytes. Inflamm. Res. 64(11), 917-928 (2015).

13. Maysinger D, Ji J, Moquin A et al. Dendritic polyglycerol sulfates in the prevention of synaptic loss and mechanism of action on glia. ACS Chem. Neurosci. 9(2), 260-271 (2018).

14. Mottas I, Milosevic A, Petri-Fink A, Rothen-Rutishauser B, Bourquin C. A rapid screening method to evaluate the impact of nanoparticles on macrophages. Nanoscale 9(7), 2492-2504 (2017)

15. Jha MK, Lee S, Park DH et al. Diverse functional roles of lipocalin-2 in the central nervous system. Neurosci. Biobehav. Rev. 49, 135-156 (2015).

16. Suk K. Lipocalin-2 as a therapeutic target for brain injury: an astrocentric perspective. Prog. Neurobiol. 144, 158-172 (2016).

- Review of the role of LCN2 secretion by astrocytes in inflammation.

17. Roudkenar MH, Kuwahara Y, Baba T et al. Oxidative stress induced lipocalin 2 gene expression: addressing its expression under the harmful conditions. J. Radiat. Res. 48(1), 39-44 (2007).

18. Moschen AR, Adolph TE, Gerner RR, Wieser V, Tilg H. Lipocalin-2: a master mediator of intestinal and metabolic inflammation. Trends Endocrinol. Metabolism 28(5), 388-397 (2017).

19. Mesquita SD, Ferreira AC, Falcao AM et al. Lipocalin 2 modulates the cellular response to amyloid beta. Cell Death Differ. 21(10), 1588-1599 (2014).

20. Kang SS, Ren Y, Liu CC et al. Lipocalin-2 protects the brain during inflammatory conditions. Mol. Psychiatry 23(2), $344-350$ (2017).

- A report on the protective role of LCN2 during septic challenge.

21. Ip JP, Nocon AL, Hofer MJ, Lim SL, Muller M, Campbell IL. Lipocalin 2 in the central nervous system host response to systemic lipopolysaccharide administration. J. Neuroinflam. 8, 124 (2011).

22. Chen Z, Jalabi W, Shpargel KB et al. Lipopolysaccharide-induced microglial activation and neuroprotection against experimental brain injury is independent of hematogenous TLR4. J. Neurosci. 32(34), 11706-11715 (2012). 
23. Zhao P, Stephens JM. STAT1, NF-кB and ERKs play a role in the induction of lipocalin-2 expression in adipocytes. Mol. Metabol. 2(3), 161-170 (2013).

24. Jung $\mathrm{M}$, Weigert $\mathrm{A}$, Tausendschon $\mathrm{M}$ et al. Interleukin-10-induced neutrophil gelatinase-associated lipocalin production in macrophages with consequences for tumor growth. Mol. Cell. Biol. 32(19), 3938-3948 (2012).

25. Boutej H, Rahimian R, Thammisetty SS, Beland LC, Lalancette-Hebert M, Kriz J. Diverging mRNA and protein networks in activated microglia reveal SRSF3 suppresses translation of highly upregulated innate immune transcripts. Cell Rep. 21(11), 3220-3233 (2017).

26. Deczkowska A, Amit I, Schwartz M. Microglial immune checkpoint mechanisms. Nat. Neurosci. 21(6), 779-786 (2018).

27. Kirkley KS, Popichak KA, Afzali MF, Legare ME, Tjalkens RB. Microglia amplify inflammatory activation of astrocytes in manganese neurotoxicity. J. Neuroinflamm. 14(1), 99 (2017).

- A report on the exacerbation of astrocyte activation due to the presence of microglia secreted factor.

28. Liddelow SA, Guttenplan KA, Clarke LE et al. Neurotoxic reactive astrocytes are induced by activated microglia. Nature 541(7638), 481-487 (2017).

29. Liddelow SA, Barres BA. Reactive astrocytes: production, function, and therapeutic potential. Immunity 46(6), 957-967 (2017).

30. Lalancette-Hebert M, Phaneuf D, Soucy G, Weng YC, Kriz J. Live imaging of toll-like receptor 2 response in cerebral ischaemia reveals a role of olfactory bulb microglia as modulators of inflammation. Brain 132, 940-954 (2009).

31. Maysinger D, Behrendt M, Lalancette-Herbert M, Kriz J. Real-time imaging of astrocyte response to quantum dots: In vivo screening model system for biocompatibility of nanoparticles. Nano Lett. 7(8), 2513-2520 (2007).

32. Hutter E, Boridy S, Labrecque S et al. Microglial response to gold nanoparticles. Acs Nano 4(5), 2595-2606 (2010).

33. Lalancette-Hebert M, Moquin A, Choi AO, Kriz J, Maysinger D. Lipopolysaccharide-QD micelles induce marked induction of TLR2 and lipid droplet accumulation in olfactory bulb microglia. Mol. Pharmaceut. 7(4), 1183-1194 (2010).

34. Kriz J, Lalancette-Hebert M. Inflammation, plasticity and real-time imaging after cerebral ischemia. Acta Neuropathol. 117(5), 497-509 (2009).

35. Lalancette-Hebert M, Faustino J, Thammisetty SS, Chip S, Vexler ZS, Kriz J. Live imaging of the innate immune response in neonates reveals differential TLR2 dependent activation patterns in sterile inflammation and infection. Brain Behav. Immun. 65, 312-327 (2017).

36. Cordeau P, Lalancette-Hebert M, Weng YC, Kriz J. Live Imaging of neuroinflammation reveals sex and estrogen effects on astrocyte response to ischemic injury. Stroke 39(3), 935-942 (2008).

37. Turk H, Haag R, Alban S. Dendritic polyglycerol sulfates as new heparin analogues and potent inhibitors of the complement system. Bioconjugate Chem. 15(1), 162-167 (2004).

38. Dagher NN, Najafi AR, Kayala KMN et al. Colony-stimulating factor 1 receptor inhibition prevents microglial plaque association and improves cognition in 3xTg-AD mice. J. Neuroinflamm. 12, 139 (2015).

39. Ramsden L, Rider CC. Selective and differential binding of interleukin (Il)-1-alpha, Il-1-beta, Il-2 and Il-6 to glycosaminoglycans. Eur. J. Immunol. 22(11), 3027-3031 (1992).

40. Mummery RS, Rider CC. Characterization of the heparin-binding properties of IL-6. J. Immunol. 165(10), 5671-5679 (2000).

41. Di Penta A, Moreno B, Reix S et al. Oxidative stress and proinflammatory cytokines contribute to demyelination and axonal damage in a cerebellar culture model of neuroinflammation. PLoS ONE 8(2), e54722 (2013).

42. Kim ID, Lee H, Kim SW et al. Alarmin HMGB1 induces systemic and brain inflammatory exacerbation in post-stroke infection rat model. Cell Death Dis. 9(4), 426 (2018).

43. Shahripour RB, Harrigan MR, Alexandrov AV. N-acetylcysteine (NAC) in neurological disorders: mechanisms of action and therapeutic opportunities. Brain Behav. 4(2), 108-122 (2014).

44. Oishi K, Hamaguchi Y, Matsushita T et al. A crucial role of L-selectin in C protein-induced experimental polymyositis in mice. Arthritis Rheumatol. 66(7), 1864-1871 (2014).

45. Xiao X, Yeoh BS, Vijay-Kumar M. Lipocalin 2: an emerging player in iron homeostasis and inflammation. Annu. Rev. Nutr. 37, 103-130 (2017).

46. Berezovskaya O, Maysinger D, Fedoroff S. The hematopoietic cytokine, colony-stimulating factor-1, is also a growth-factor in the Cns congenital absence of Csf-1 in mice results in abnormal microglial response and increased neuron vulnerability to injury. Int. J. Dev. Neurosci. 13(3-4), 285-299 (1995).

47. Elmore MRP, Najafi AR, Koike MA et al. Colony-stimulating factor 1 receptor signaling is necessary for microglia viability, unmasking a microglia progenitor cell in the adult brain. Neuron 82(2), 380-397 (2014).

48. Berg D, Gerlach M, Youdim MBH et al. Brain iron pathways and their relevance to Parkinson's disease. J. Neurochem. 79(2), 225-236 (2001).

49. Bishop GM, Robinson SR, Liu Q, Perry G, Atwood CS, Smith MA. Iron: A pathological mediator of Alzheimer disease? Dev. Neurosci. (Basel) 24(2-3), 184-187 (2002). 
50. Moss DW, Bates TE. Activation of murine microglial cell lines by lipopolysaccharide and interferon-gamma causes NO-mediated decreases in mitochondrial and cellular function. Eur. J. Neurosci. 13(3), 529-538 (2001).

51. Wilkins HM, Weidling IW, Ji Y, Swerdlow RH. Mitochondria-derived damage-associated molecular patterns in neurodegeneration. Front. Immunol. 8, 508 (2017).

52. Zhang Q, Raoof M, Chen Y et al. Circulating mitochondrial DAMPs cause inflammatory responses to injury. Nature 464(7285), 104-U115 (2010).

53. Matzinger P. Tolerance, danger, and the extended family. Annu. Rev. Immunol. 12, 991-1045 (1994).

54. Hanamsagar R, Hanke ML, Kielian T. Toll-like receptor (TLR) and inflammasome actions in the central nervous system. Trends Immunol. 33(7), 333-342 (2012).

55. Mangan MSJ, Olhava EJ, Roush WR, Seidel HM, Glick GD, Latz E. Targeting the NLRP3 inflammasome in inflammatory diseases. Nat. Rev. Drug Discov. 17(8), 588-606 (2018).

56. Shen Y, Qin H, Chen J et al. Postnatal activation of TLR4 in astrocytes promotes excitatory synaptogenesis in hippocampal neurons. J. Cell Biol. 215(5), 719-734 (2016).

57. Vaure C, Liu YQ. A comparative review of toll-like receptor 4 expression and functionality in different animal species. Front. Immunol. 5, 316 (2014).

58. Qanungo S, Wang M, Nieminen A-L. N-Acetyl-l-cysteine enhances apoptosis through inhibition of nuclear factor- $\mathrm{kB}$ in hypoxic murine embryonic fibroblasts. J. Biol. Chem. 279(48), 50455-50464 (2004).

59. Dekhuijzen PNR. Antioxidant properties of N-acetylcysteine: their relevance in relation to chronic obstructive pulmonary disease. Eur. Respir. J. 23(4), 629-636 (2004).

60. Minogue AM, Barrett JP, Lynch MA. LPS-induced release of IL-6 from glia modulates production of IL-1 beta in a JAK2-dependent manner. J. Neuroinflamm. 9, 126 (2012).

61. Boche D, Perry VH, Nicoll JAR. Review: Activation patterns of microglia and their identification in the human brain. Neuropath. Appl. Neuro. 39(1), 3-18 (2013).

62. Poli M, Girelli D, Campostrini N et al. Heparin: a potent inhibitor of hepcidin expression in vitro and in vivo. Blood 117(3), 997-1004 (2011). 